\title{
Influence of Perylene Doping on Performance of Organic Solar Cells Based on P3HT:PCBM Blend
}

\author{
Yanhui Lou*, Zhaokui Wang, Shigeki Naka and Hiroyuki Okada \\ Graduate School of Science and Engineering, University of Toyama, \\ Gofuku 3190, Toyama 930-8555, Japan \\ *E-mail:yanhuilou@hotmail.co.jp
}

Keyword: bulk heterojunciton, polymer solar cells, small molecular, dye doping

\section{Introduction}

Organic solar cells (OSCs) have attracted more attention due to their potential advantages such as low cost, light weight and possible fabricating on flexible substrate [1]. Almost $8 \%$ power conversion efficiencies for OSCs have already been achieved with growing tendency [2]. However, the efficiency of OSCs is still significantly lower than that of other inorganic solar cells, which prevents practical applications in large scale [3].

Two major factors that restrict the further enhancement of the power conversion efficiency are of short exciton diffusion length and poor charge carrier mobility in most organic semiconductors. Thereafter, blending electron donor and acceptor known as bulk heterojunctions (BHJs) structure is proposed for addressing the first problem [4]. In addition, tandem structures, in which multiple organic layers covering complementary absorption spectra are used for enhancing spectral coverage, are designed for improving device efficiency $[5,6]$. However, the complicated fabrication processes and the demand for the intercellular layer are additional difficulties.

The combination of poly(3-hexylthiophene) (P3HT) and [6,6]-phenyl C61-butyric acid methyl ester (PCBM) in organic blends has recently shown high photovoltaic performance $[7,8]$. However, most of previous studies using P3HT and PCBM have been limited to their binary blends, even though a couple of reports have been out for ternary blend solar cells by introducing semiconducting polymers in order to tune the performance of OSCs $[9,10]$. Up to date, no study has been so far reported on the attempt to dope small molecule dyes into the P3HT:PCBM blends. In present work, we attempt to dope small molecule dyes, 5,6,11,12-tetraphenylnaphthacene (rubrene), quinacridone (QD) and perylene, which are well-known dopant luminance materials for organic light emitting devices (OLEDs)and have good solubility in organic solvents, into the P3HT:PCBM blends for investigating the performance of OSCs. It will be expected to seek for a suitable small molecule dye which can improve the device performance and the nature of performance improvement will be discussed briefly.

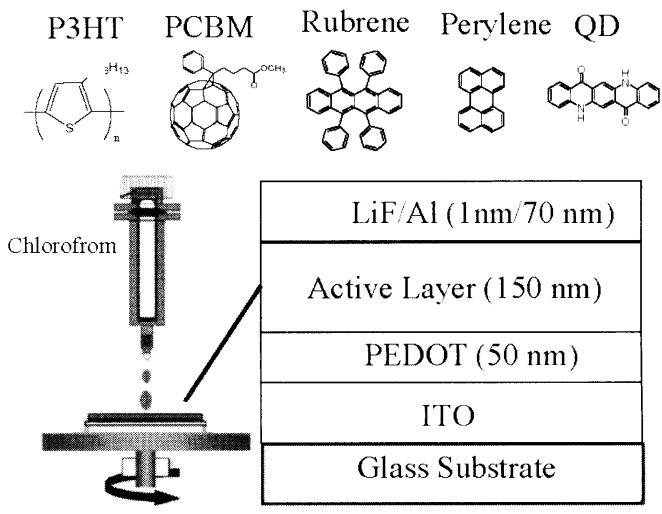

Figure 1. the device structure of the polymer solar cells and the molecular structures of the organic materials used upon investigation. 


\section{Experimental}

All the devices were fabricated on patterned indium tin oxide (ITO) coated glass substrates, which had been carefully cleaned by standard procedure. After the cleaned ITO substrates were exposed to UV-ozone, a thin layer of poly(3,4-ethylenedioxythiophene):poly(styrenesulfonate) (PEDOT:PSS) was spin-coated onto the ITO surface with a speed of $3000 \mathrm{rpm}$ (round per minute) for $60 \mathrm{~s}$ and then baked at $200 \circ \mathrm{C}$ for $5 \mathrm{~min}$. The active layer of P3HT:PCBM (dissolved in chloroform with a weight ratio of $1: 1$ ) with and without small molecule dyes (dissolved in chloroform) doping were both spin-coated at $1000 \mathrm{rpm}$. Small molecule dyes, 5,6,11,12-tetraphenylnaphthacene (rubrene), quinacridone (QD) and perylene were used as dopant. After baking at $150{ }^{\circ} \mathrm{C}$ for $10 \mathrm{~min}, \mathrm{LiF}$ and $\mathrm{Al}$ cathode were deposited via thermal evaporation through a shadow mask, giving an active device area of $0.04 \mathrm{~cm}^{2}$. Figure 1 shows the device structure of the polymer solar cells and the molecular structures of the organic materials used in the study. The current-voltage characteristics were measured using a semiconductor parameter analyzer (HP 4155B) under a simulated air mass (AM) $1.5 \mathrm{G}$ spectrum illumination. The absorption spectra are measured by spectrophotometer (Hitachi 330).

\section{Results and Discussion}

3.1. Different dye doping

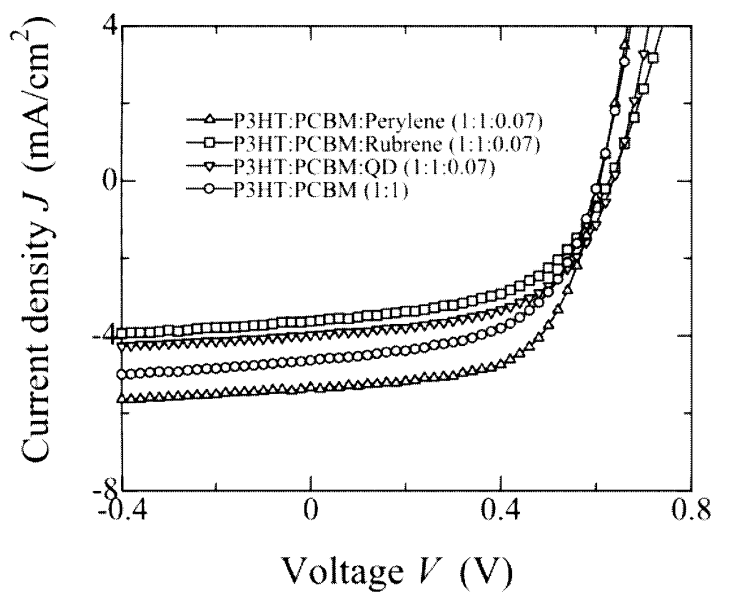

Figure 2. $J-V$ characteristics of the standard device and different dye doped devices under illumination.
The current density versus voltage $(J-V)$ characteristics of the standard device: ITO/PEDOT/P3HT:PCBM $(1: 1) / \mathrm{LiF} / \mathrm{Al}$ and dye doped device: ITO/ PEDOT/ P3HT:PCBM:dye $(1: 1: 0.07) / \mathrm{LiF} / \mathrm{Al}$ under illumination at $150 \mathrm{~mW} / \mathrm{cm}^{2}$ are depicted in Figure 2. And the detailed performance parameters including short-circuit current density $\left(J_{s c}\right)$, open-circuit voltage $\left(V_{o c}\right)$, fill factor $(F F)$ and power conversion efficiency $(P C E)$ are listed in Table 1.

Table 1. Performance summary (including $J_{s c}, V_{o c}$. $F F$ and $P C E$ ) of standard and different dye doped OSCs.

\begin{tabular}{lllll}
\hline Dopant & $\begin{array}{c}J_{s c} \\
\left(\mathrm{~mA} / \mathrm{cm}^{2}\right)\end{array}$ & $\begin{array}{l}V_{o c} \\
(\mathrm{~V})\end{array}$ & $\begin{array}{l}F F \\
(\%)\end{array}$ & $\begin{array}{l}P C E \\
(\%)\end{array}$ \\
\hline No doping & 4.64 & 0.61 & 54 & 1.55 \\
Perylene & 5.34 & 0.62 & 60 & 1.97 \\
Rubrene & 3.63 & 0.64 & 52 & 1.20 \\
QD & 3.98 & 0.64 & 55 & 1.39 \\
\hline
\end{tabular}

It is obvious that $J_{s c}$ is enhanced from $4.64 \mathrm{~mA} / \mathrm{cm}^{2}$ to $5.34 \mathrm{~mA} / \mathrm{cm}^{2}$ after doping perylene into blending films, resulting in improvement of $P C E$ from $1.55 \%$ to $1.97 \%$ although no change of $V_{o c}$. While, for Rubrene and QD doped devices, $J_{s c}$ is largely decreased although $V_{o c}$ is slightly increased, finally resulting in a worse $P C E$.

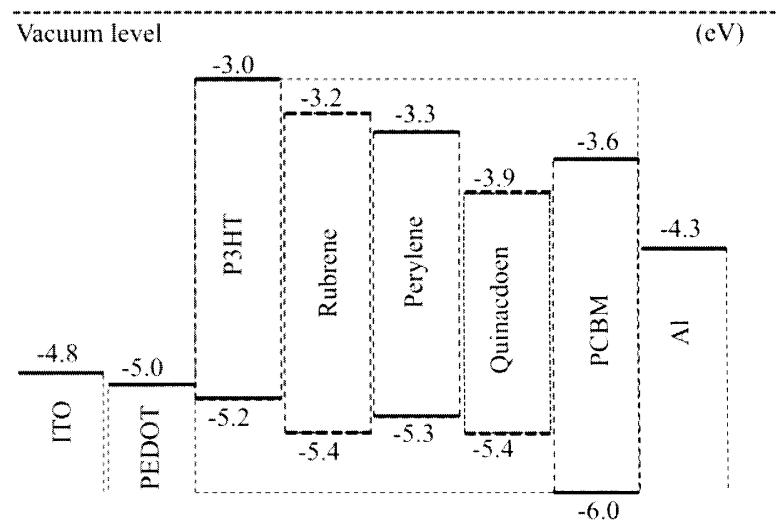

Figure 3. Energy levels of organic materials upon investigation.

Up to now, the origin of $V_{o c}$ remains still controversial. In general, it is regarded that $V_{o c}$ depends mainly on the energy difference between the highest occupied molecular orbital (HOMO) of the donor and the lowest 
unoccupied molecular orbital (LUMO) of the acceptor $[11,12]$. Several groups reported that $V_{o c}$ is improved by doping Rubrene into small molecular $\mathrm{CuPc} / \mathrm{C}_{60}$ OSCs [12-14]. They attributed the increased $V_{o c}$ to the large energy difference between the HOMO of Rubrene and LUMO of $\mathrm{C}_{60}$. In present study, the energy levels of all organic materials are shown in Figure 3. From the energy difference between the HOMO of dyes and LUMO of PCBM compared with that between P3HT and PCBM, the changes of $V_{o c}$ of devices with and without dye doping as mentioned above is easily understood.

Moreover, we measured absorption spectra of dye doped- and un-doped P3HT:PCBM blending films for clarifying the mechanism of large changes of device performance with and without different dye doping. The typical results are shown in Figure 4. The main absorption peaks of pure perylene, rubrene and QD are located in the vicinities of $440 \mathrm{~nm}, 300 \mathrm{~nm}$ and $480 \mathrm{~nm}$, respectively. It is found that the complementary absorption of perylene and QD enhanced the light harvesting in a range of 400-600 nm, thereby broadending the absorption spectrum of the whole blending films. For rubrene doped blending films, the slight absorption enhancement is also observed in the vicinity of $320 \mathrm{~nm}$. Just from the analysis of absorption spectrum, the performance of rubrene or QD doped devices should also be improved comparing with undoped devices. While, the device performance is deteriorated after doping rubrene or QD into P3HT: PCBM blending films as above mentioned. It is implied that there are some other reasons for the lowering of device performance after doping rubrene or QD into P3HT: PCBM blending films.

In rubrene doped P3HT: PCBM blending based OSCs, the generated holes and electrons from excitons is supposed to be captured by Rubrene molecules due to its dual nature of acting as electron hopping site and hole trap site, which decreases the carriers diffusing to electrodes and resulting in lower device performance. In addition, it can be seen that QD has a narrow band gap (1.5 $\mathrm{eV}$ ) and the HOMO and LUMO of QD are almost positioned between the HOMOs and LUMOs of P3HT and PCBM. It is assumed that carrier capturing by QD molecules was also occurred in
QD doped P3HT: PCBM blending based OSCs and resulting in a low device performance compared with that of undoped devices.

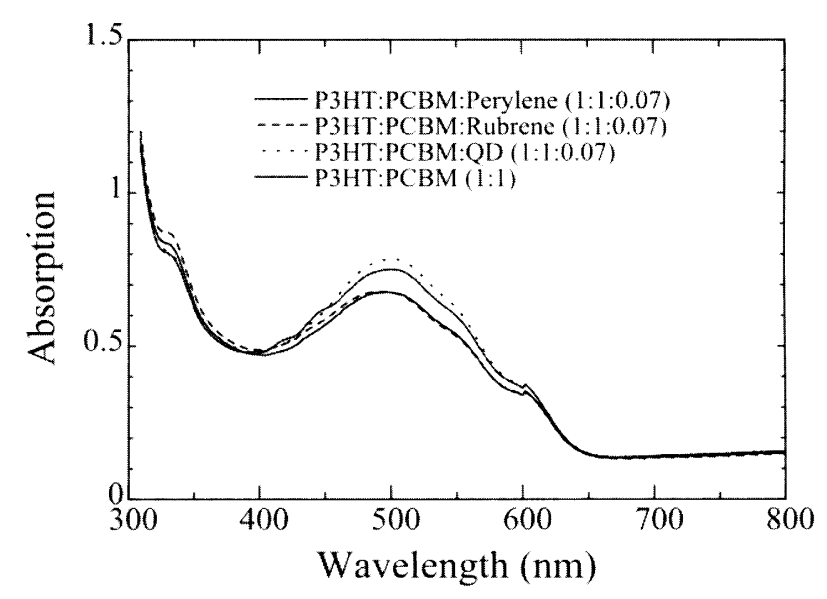

Figure 4. Optical absorption of P3HT: PCBM blending films with and without different dye doping.

\subsection{Optimization of perylene doping ratio}

The doping ratio of perylene is varied in the range of $0-0.1 \mathrm{wt} \%$ in order to elucidate the effect of perylene doping ratio on the performance of P3HT: PCBM blending based OSCs. The illuminated $J-V$ characteristics of the devices with different perylene doping ratio are depicted in Figure 5 and the key cell parameters of OSCs as a function of perylene doping ratio are listed in Table 2.

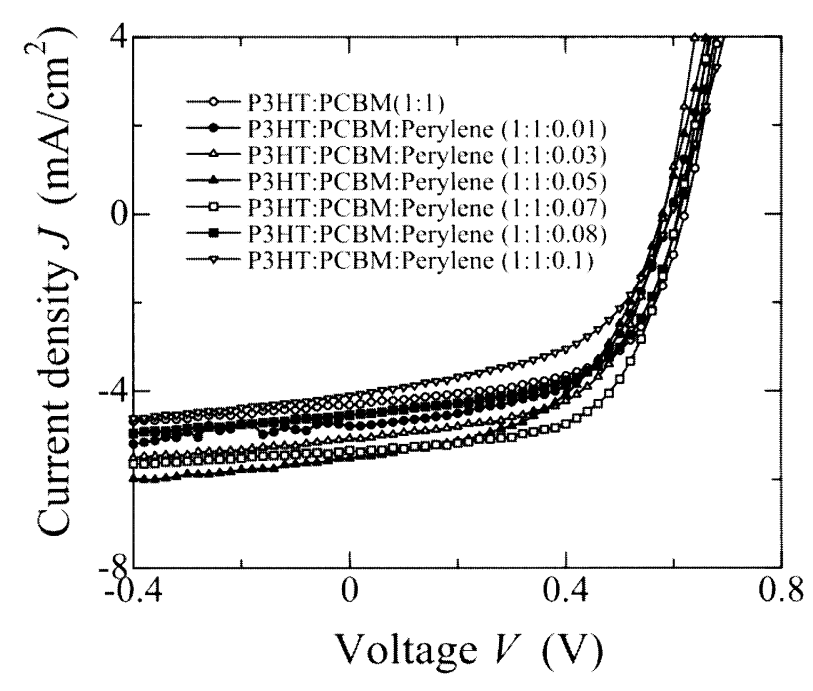

Figure 5. $J-V$ characteristics of the standard device and perylene doped devices with different doping ratio under illumination. 
Table 2. Performance summary (including $J_{s c}, V_{o c}$, $F F$ and $P C E$ ) of standard and perylene doped OSCs with different doping ratio.

\begin{tabular}{ccccc}
\hline $\begin{array}{c}\text { Perylene } \\
\text { ratio }\end{array}$ & $\begin{array}{c}J_{s c} \\
\left(\mathrm{~mA} / \mathrm{cm}^{2}\right)\end{array}$ & $\begin{array}{l}V_{o c} \\
(\mathrm{~V})\end{array}$ & $\begin{array}{c}F F \\
(\%)\end{array}$ & $\begin{array}{l}P C E \\
(\%)\end{array}$ \\
\hline 0 & 4.64 & 0.61 & 54 & 1.55 \\
0.01 & 4.79 & 0.59 & 56 & 1.58 \\
0.03 & 5.07 & 0.59 & 58 & 1.72 \\
0.05 & 5.52 & 0.57 & 51 & 1.62 \\
0.07 & 5.34 & 0.62 & 60 & 1.97 \\
0.08 & 4.55 & 0.61 & 59 & 1.58 \\
0.10 & 4.11 & 0.59 & 51 & 1.23 \\
\hline
\end{tabular}

It is found that the PCE of OSCs can be improved after doping perylene into P3HT: PCBM blending films with different doping ratio excluding a higher doping ratio $(0.10 \mathrm{wt} \%)$. With $0.10 \mathrm{wt} \%$ doping ratio, $J_{s c}$ is decreased from $4.64 \mathrm{~mA} / \mathrm{cm}^{2}$ to $4.11 \mathrm{~mA} / \mathrm{cm}^{2}$ and $V_{o c}$ is also decreased from $0.61 \mathrm{~V}$ to $0.59 \mathrm{~V}$ compared with undoped device, which results in a lower $P C E$ with a deteriorated $F F$. By doping 0.07 $\mathrm{wt} \%$ perylene into $\mathrm{P} 3 \mathrm{HT}$ : PCBM blending films, $J_{s c}$ is increased to $5.34 \mathrm{~mA} / \mathrm{cm}^{2}$ and $V_{o c}$ is slightly increased, which results in a large $P C E(1.97 \%)$ with a good $F F$. The effects of perylene doping ratio on device performance of P3HT: PCBM blending based OSCs is not well understood presently and further investigation such as measurement of absorption spectra is necessary in the future.

\section{Conclusion}

We have investigated the doping influence of different small molecular dyes on the performance of P3HT: PCBM blending based OSCs. For the perylene-doped device, the improved performance may be due to long wave absorption peak $(440 \mathrm{~nm})$ of perylene, resulting in an enhanced optical absorption at long wave range for doped P3HT: PCBM blending films. In addition, a maximum $1.97 \%$ power conversion efficiency is obtained after optimizing the perylene doping ratio with $0.07 \mathrm{wt} \%$.

\section{References}

1. Y. J. He, H. Y. Chen, J. H. Hou, and Y. F. Li, J. Am. Chem. Soc., 132 (2010), 1377.

2. M. A. Green, K. Emery, Y. Hishikawa, and W. Warta, Prog. Photovolt.: Res. Appl., 18 (2010), 144.

3. Y. Y. Liang, Adv. Mater., 22 (2010), E135.

4. G. Yu, J. Gao, J. C. Hummelen, F. Wudl and A. J. Heeger, Science, 270 (1995), 1789.

5. J. Y. Kim, K. Lee, N. E. Coates, D. Mose, T. Q. Nguyen, M. Dante, and J. A. Heeger, Science, 317 (2007), 222.

6. D. W. Zhao, X. W. Sun, C. Y. Jiang, A. K. K. Kyaw, G. Q. Lo, and D. L. Kwong, Appl. Phys. Lett., 93 (2008), 083305.

7. W. Ma, C. Yang, X. Gong, K. -S. Lee, A. J Heeger, Adv. Funct. Mater., 15 (2005), 1617.

8. V. D. Mihailetchi, H. Xie, B. de Boer, L. J. A. Koster, P. W. M. blom, Adv. Funct. Mater., 16 (2007), 699.

9. Y. Kim, M. Shin, H. Kim, Y. Ha, and C. S. Ha, J. Phys. D: Appl. Phys. 41 (2008), 225101.

10. H. Kim, M. Shin, and Y. Kim, J. Phys. Chem. C, 113 (2009), 1620.

11. P. C. Kao, S. Y. Chu, H. H. Huang, Z. L. Tseng, and Y. C. Chen, Thin Solid Films 517 (2009), 5301.

12. M. C. Scharber, D. Mühlbacher, M. Koppe, P. Denk, C. Waldauf, A. J. Heeger, and C. J. Brabec, Adv. Mater, 18 (2006), 789.

13. Y. Hayashi, H. Sakuragi, T. Soga, I. Alexandrou, G. A. J. Amaratunga, Colloids and Surfaces A, 313-314 (2008), 422.

14. M. Y. Chan, S. L. Lai, M. K. Fung, C. S. Lee, and S. T. Lee, Appl. Phys. Lett., 90 (2007), 023504 